\title{
Subband Array Implementations for Space-Time Adaptive Processing
}

\author{
Yimin Zhang \\ Center for Advanced Communications, Villanova University, Villanova, PA 19085, USA \\ Email:yimin@ieee.org
}

Kehu Yang

Electronic Engineering Research Institute, School of Electronic Engineering, Xidian University, Xi'an, Shaanxi 710071, China

Email:yang001@xidian.edu.cn

\author{
Moeness G. Amin \\ Center for Advanced Communications, Villanova University, Villanova, PA 19085, USA \\ Email:moeness.amin@villanova.edu
}

Received 1 January 2004; Revised 30 June 2004

\begin{abstract}
Intersymbol interference (ISI) and cochannel interference (CCI) are two primary sources of signal impairment in mobile communications. In order to suppress both ISI and CCI, space-time adaptive processing (STAP) has been shown to be effective in performing spatio-temporal equalization, leading to increased communication capacity as well as improved quality of service. The high complexity and slow convergence, however, often impede practical STAP implementations. Several subband array structures have been proposed as alternatives to STAP. These structures provide optimal or suboptimal steady-state performance with reduced implementation complexity and improved convergence performance. The purpose of this paper is to investigate the steady-state performance of subband arrays with centralized and localized feedback schemes, using different decimation rates. Analytical expressions of the minimum mean-square error (MMSE) performance are derived. The analysis assumes discrete Fourier transform (DFT)-based subband arrays and considers both unconstrained and constrained weight adaptations.
\end{abstract}

Keywords and phrases: space-time adaptive processing, subband array, array processing, mobile communications, intersymbol interference, cochannel interference.

\section{INTRODUCTION}

The applications of wireless communications are rapidly expanding from voice transmission to a wide class of multimedia information. With such increasing needs, wireless communication systems are developing toward higher-speed digital wireless networks. The communication channels are often frequency selective, as a result of long multipath delays relative to the symbol period, causing intersymbol interference (ISI). In many mobile communication systems, where the frequency resource is reused, cochannel interference (CCI) represents another source of channel distortion and signal impairment. Therefore, ISI and CCI are two primary sources that limit the communication capacity and the quality of services in mobile communications.

While adaptive arrays are effective for spatial processing of CCI suppression; whereas adaptive equalizers are effective for temporal filtering for ISI reduction, neither of them are effective when both the CCI and ISI are present.
The use of space-time adaptive processing (STAP) technology is an effective way to perform spatio-temporal equalization that mitigates the above two problems $[1,2]$. Objectives are to increase the communication capacity and enhance the quality of services. A variety of algorithms have been developed for the implementation of the STAP systems, including those based on least-mean square (LMS), recursive least squares (RLS), and sample matrix inversion (SMI). The direct use of STAP system often involves high-dimension space in the joint spatial and temporal domain. This, in turn, brings a high complexity and slow convergence rate, rendering the STAP system unattractive. This shortcoming has motivated extensive research work for devising alternative implementation $[3,4,5,6,7,8,9]$. Among those methods, subband or frequency-domain arrays offer the amenability of parallel implementation with reduced processing rates in each subband $[10,11]$. With appropriate power normalization or data self-orthogonalization, subband arrays can achieve improved convergence [12, 13, 14]. 
The subband (including frequency-domain) adaptive arrays can be classified, in terms of their feedback methods, into two classes, namely, centralized feedback and localized feedback. In [7], the partial feedback scheme was also introduced as a generalization of the above schemes. For the centralized feedback schemes, Compton has shown that the frequency-domain array provides identical steady-state performance of the corresponding STAP system [15]. Such equivalence, however, is valid only for the undecimated (window sliding) cases. The use of decimation may provide significant system complexity reduction in subband array implementations. The analysis of the performance degradation with the use of decimation has been recently considered by Tran et al. [8] only for ISI without taking the CCI signals into account. On the other hand, for the localized and partial feedback schemes, low computations, parallel processing, and faster convergence can be achieved at the cost of suboptimal steady-state performance $[3,7]$. Although the investigation of localized subband arrays, according to Compton [15], dated back to early 1970s [16], a detailed performance analysis, to our knowledge, was not available until recently $[7,8]$. In [7], the performance of discrete Fourier transform (DFT) filter bank-based subband arrays has been considered for the aforementioned three feedback schemes where no decimation is applied. In [8], the performance of localized feedback subband array is analyzed for the DFT-based subband arrays in the absence of CCI users. The results of [8] show that, in a frequency-selective multipath fading environment, the subband array performance depends on the number of subbands, input signal-to-noise ratio (SNR), the source directions-of-arrival (DOAs), and the multipath time delays. In addition to the above literature, [3] provides various numerical comparison results between the centralized and localized feedback schemes.

In this paper, we investigate the performance of DFTbased subband arrays with different decimation rates. Both unconstrained and constrained subband array structures are considered. To consider the minimum mean-square error (MMSE) performance, the reference signal is considered to be available. The steady-state performances of subband adaptive arrays with the centralized and localized feedback schemes and different decimation rates are analyzed, and expressions for the MMSE are derived. It is shown that decimation compromises the optimum performance for both centralized and localized feedback subband array schemes. The convergence performance of different subband array structures is also investigated and compared.

It is worth noting that there is an extensive literature in frequency-domain equalizations and echo-cancellation methods using single-sensor receivers (see, e.g., $[17,18,19$, $20]$ and references therein). These methods provide a fundamental development in the theory of subband processing. However, important differences exist between singleand multi-sensor systems in both formulations and performances. The inclusion of the spatial domain to subband signal processing affects both the processing structure and the performances. Single-antenna receivers cannot deal with the cancellation of CCIs. In addition, we specifically address the problem of subband arrays with arbitrary decimation rates for both centralized and localized feedback structures.

The rest of this paper is organized as follows. Section 2 introduces the signal model and reviews the analysis of STAP performance. Section 3 considers the subband decomposition, and the aliasing issue with the use of decimation. Section 4 formulates the subband arrays with both centralized and localized feedback schemes. The steady-state performance of different subband array structures is analyzed in Section 5. Section 6 compares the computational complexity between the subband arrays and conventional STAP systems. Section 7 considers the convergence performance where data self-orthogonalization and the step-size selection are addressed. Numerical examples are provided in Section 8 for illustration.

\section{SPACE-TIME ADAPTIVE PROCESSING}

\subsection{Signal model}

We consider a base station using an antenna array of $N$ sensors with $P$ users. Without loss of generality, the user signal of interest is denoted as $s_{1}(n)$. The signals from other users as $s_{p}(n), p=2, \ldots, P$, form the CCIs to the signal of interest. When frequency-selective channels are considered for each user, the received data vector at the array is expressed as

$$
\begin{aligned}
\underline{\mathbf{x}}(t) & =\left[x_{1}(t), \ldots, x_{N}(t)\right]^{\mathrm{T}} \\
& =\sum_{p=1}^{P} \sum_{i=-\infty}^{\infty} s_{p}(i) \underline{\mathrm{h}}_{p}(t-i T)+\underline{\mathrm{b}}(t),
\end{aligned}
$$

where the superscript $\mathrm{T}$ denotes matrix or vector transpose, $s_{p}(n)$ and $\underline{\mathrm{h}}_{p}(t)$ are the $n$th information symbol and the channel response vector (including the pulse shaping) of the $p$ th user, respectively, and $\underline{\mathrm{b}}(t)$ is the additive noise vector.

The data vector is sampled at $t=n T+i \Delta$, where $T$ is the symbol duration of the signal waveform and $\Delta$ is the sampling period. The integer ratio of $J=T / \Delta$ is referred to as the oversampling factor. Then, the data vector takes the following discrete-time expression:

$$
\underline{\mathrm{x}}(n T+i \Delta)=\sum_{p=1}^{P} \sum_{d=0}^{\infty} s_{p}(n-d) \underline{\mathrm{h}}_{p}(d T+i \Delta)+\underline{\mathrm{b}}(n T+i \Delta) .
$$

We make the following assumptions.

(A1) The time required for the received waveform associated with a given transmission path to propagate across the array is much smaller than the inverse of the user signal bandwidth.

(A2) The user signals $s_{p}(n), p=1,2, \ldots, P$, are widesense stationary (if sampled at the symbol rate, i.e., $J=1$ ) or cyclostationary (if sampled at fractionally spaced symbol cycle, i.e., $J>1$ ). These signals are independent and identically distributed (i. i. d.) with $E\left[s_{p}(n) s_{p}^{*}(n)\right]=1$, where $E(\cdot)$ denotes the statistical expectation operator and the superscript $*$ denotes the complex conjugate. 
(A3) All channels $\underline{\mathrm{h}}_{p}(t), p=1,2, \ldots, P$, are linear timeinvariant, and of a finite duration within $\left[0,\left(D_{p}+1\right) T\right]$, where $D_{p}$ are nonnegative integers.

(A4) The noise vector $\underline{b}(n)$ is zero-mean and temporally and spatially white with variance $\sigma$ at each array sensor.

Under these assumptions, we can stack the $J$ samples within each symbol period resulting in the following $N J \times 1$ vector containing data received at the $N J$ virtual channels (or extended channels):

$$
\begin{aligned}
\tilde{\mathbf{x}}(n) & =\left[\begin{array}{llll}
\underline{\mathbf{x}}^{\mathrm{T}}[n T] & \underline{\mathbf{x}}^{\mathrm{T}}[n T-\Delta] & \cdots & \underline{\mathbf{x}}^{\mathrm{T}}[n T-(J-1) \Delta]
\end{array}\right]^{\mathrm{T}} \\
& =\sum_{p=1}^{P} \sum_{d=0}^{D_{p}} s_{p}(n-d) \tilde{\mathbf{h}}_{p}(d)+\tilde{\mathbf{b}}(n),
\end{aligned}
$$

where

$$
\begin{aligned}
\tilde{\mathbf{h}}_{p}(n) & =\left[\begin{array}{llll}
\underline{\mathrm{h}}_{p}^{\mathrm{T}}[n T] & \underline{\mathrm{h}}_{p}^{\mathrm{T}}[n T-\Delta] & \cdots & \underline{\mathrm{h}}_{p}^{\mathrm{T}}[n T-(J-1) \Delta
\end{array}\right]^{\mathrm{T}}, \\
\tilde{\mathbf{b}}(n) & =\left[\begin{array}{llll}
\underline{\mathrm{b}}^{\mathrm{T}}[n T] & \underline{\mathrm{b}}^{\mathrm{T}}[n T-\Delta] & \cdots & \underline{\mathrm{b}}^{\mathrm{T}}[n T-(J-1) \Delta]
\end{array}\right]^{\mathrm{T}} .
\end{aligned}
$$

\subsection{Space-time adaptive processing}

When a $J M$-tap FIR filter is used at the output of each array sensor, or equivalently, an $M$-tap FIR filter is used at each of the $N J$ virtual channel, we obtain a $M N J \times 1$ vector that contains all the input values at the STAP system at time instant $n:$

$$
\mathbf{x}(n)=\left[\begin{array}{llll}
\tilde{\mathbf{x}}^{\mathrm{T}}(n) & \tilde{\mathbf{x}}^{\mathrm{T}}(n-1) & \cdots & \tilde{\mathbf{x}}^{\mathrm{T}}(n-M+1)
\end{array}\right]^{\mathrm{T}} .
$$

Similarly, we define

$$
\begin{gathered}
\mathbf{b}(n)=\left[\begin{array}{lllll}
\tilde{\mathbf{b}}^{\mathrm{T}}(n) & \tilde{\mathbf{b}}^{\mathrm{T}}(n-1) & \cdots & \tilde{\mathbf{b}}^{\mathrm{T}}(n-M+1)
\end{array}\right]^{\mathrm{T}}, \\
\mathbf{s}_{p}(n)=\left[\begin{array}{llllll}
s_{p}(n) & s_{p}(n-1) & \cdots & s_{p}\left(n-M-D_{p}\right)
\end{array}\right]^{\mathrm{T}}, \\
\mathbf{H}_{p} \\
=\left[\begin{array}{ccccccc}
\tilde{\mathbf{h}}_{p}(0) & \cdots & \tilde{\mathbf{h}}_{p}\left(D_{p}\right) & \mathbf{0} & \cdots & \cdots & \mathbf{0} \\
\mathbf{0} & \tilde{\mathbf{h}}_{p}(0) & \cdots & \tilde{\mathbf{h}}_{p}\left(D_{p}\right) & \mathbf{0} & \cdots & \mathbf{0} \\
\vdots & \ddots & & \ddots & \vdots & \ddots & \vdots \\
\mathbf{0} & \cdots & \cdots & \mathbf{0} & \tilde{\mathbf{h}}_{p}(0) & \cdots & \tilde{\mathbf{h}}_{p}\left(D_{p}\right)
\end{array}\right] .
\end{gathered}
$$

Then, we represent all $M$ symbol samples captured at the $N J$ virtual channels of the STAP as

$$
\mathbf{x}(n)=\sum_{p=1}^{P} \mathbf{H}_{p} \mathbf{s}_{p}(n)+\mathbf{b}(n) .
$$

Denote $\mathbf{w}^{*}$ as the weight vector corresponding to $\mathbf{x}(n)$. Then the output of the STAP becomes

$$
y(n)=\mathbf{w}^{\mathrm{H}} \mathbf{x}(n),
$$

where the superscript $\mathrm{H}$ denotes Hermitian (conjugate transpose) operation. When a training signal, which is an ideal replica of $s_{1}(n)$, is available at the receiver, the optimum weight vector under the MMSE criterion can be provided using the Wiener-Hopf solution:

$$
\mathbf{w}_{\mathrm{opt}}=\mathbf{R}_{o}^{-1} \mathbf{r}_{o}
$$

with

$$
\mathbf{R}_{o}=E\left[\mathbf{x}(n) \mathbf{x}^{\mathrm{H}}(n)\right], \quad \mathbf{r}_{o}=E\left[\mathbf{x}(n) s_{1}^{*}(n-v)\right],
$$

where $v$ is a delay [21], which is chosen to minimize the following MMSE:

$$
\mathrm{MMSE}=E\left|s_{1}(n-v)-\mathbf{w}_{\mathrm{opt}}^{\mathrm{H}} \mathbf{x}(n)\right|^{2}=1-\mathbf{r}_{o}^{\mathrm{H}} \mathbf{R}_{o}^{-1} \mathbf{r}_{o} .
$$

Substituting (7) in (10), and using assumption (A2), we have

$$
\mathbf{r}_{o}=\mathbf{H}_{1} \mathbf{e}_{v}
$$

where

$$
\mathbf{e}_{v}=[\underbrace{0, \ldots, 0}_{v}, 1,0, \ldots, 0]^{\mathrm{T}},
$$

provided $0<v<M+D_{1}-1$. That is, $\mathbf{r}_{o}$ is the $(v+1)$ th column of $\mathbf{H}_{1}$ [22]. For example, choosing $v=0$ or $v=M+D_{1}-1$ yields only one effective weight for each virtual channel. The optimum value of $v$ usually occurs around $\left(M+D_{1}\right) / 2-1$, but the actual result depends on the channel characteristics.

Typically, $J$ is chosen as either one or two [23]. In addition, it can be shown [21] that, when the channels meet the following conditions:

(1) $\mathbf{H}_{1}$ is full column rank,

(2) the columns of $\mathbf{H}_{1}$ are linearly independent of the columns of $\mathbf{H}_{p}, p=2, \ldots, P$,

the selection of $M$ and $N$ satisfying

$$
M N J \geq \operatorname{column} \operatorname{rank}\{\mathbf{H}\}
$$

yields perfect equalization conditions in noise-free scenarios, where $\mathbf{H}=\left[\mathbf{H}_{1}, \ldots, \mathbf{H}_{P}\right]$. When all $\mathbf{H}_{p}, p=1, \ldots, P$, are full column rank, the above requirement is equivalent to

$$
\begin{gathered}
M \geq \frac{1}{N J-P} \sum_{p=1}^{P} D_{p}, \\
N J>P .
\end{gathered}
$$

\section{SUBBAND DECOMPOSITION}

\subsection{Subband decomposition and subband arrays}

Subband decomposition and reconstruction of a signal are performed by exploiting a set of analysis and synthesis filters. The analysis filters decompose a wideband signal into a set of narrowband subband signal components [24]. Highly decorrelated subband signals are often desired in subband decomposition-based equalization problems to ensure faster 
convergence and reduce the performance loss in localized feedback schemes $[7,21,25,26]$. To achieve effective decorrelation between subband signals, the analysis filters are required to be close to the ideal bandpass filters [5, 26, 27]. This necessitates the use of long analysis filters (i.e., filters with long taps) and, therefore, is usually not desirable. Long analysis filters not only imply a long time delay in the process of subband decomposition and reconstruction of the signals, but also apply a strict condition to the stationarity of the channel. More importantly, for nonblind subband array systems, long analysis filters yield ineffective use of the training signals. For these reasons, we consider, in this paper, DFT-based filter bank, where the transform matrix of the analysis filters is square. We maintain that long analysis filters remain useful in certain application scenarios such as blind spatio-temporal equalization and echo-cancellation applications, where the training signal is not a problem.

Combining the subband signal processing and array processing results in subband array processing. So far, several subband arrays have been proposed for spatio-temporal equalizations $[3,4,5,6,7,8,9,10,12]$. For DFT-based subband arrays, the performance without decimation has been discussed in [7], whereas the performance with decimation is analyzed for CCI-free situations in [8]. In the latter, only the maximum decimation is considered, that is, the decimation rate is the same as the number of subband bins, resulting in a blockwise subband array scheme.

In this paper, we deal with more general cases of DFTbased subband arrays of arbitrary decimation rates $L$. That is, for each set of data processed in the subband array processing, $L$ output data of $y(n)$ are used. As a result, the processing window slides every $L$ symbols. It also implies that the weights are updated every $L$ symbols. The decimation rate is chosen between one (i.e., no decimation) and the number of subband bins $M$ (i.e., maximum decimation), namely, $1 \leq L \leq M$.

\subsection{Consideration of decimation}

One important issue to be considered in decimated subband signal processing is the alias problem. For simplicity of notation and explanation, we illustrate this problem by using a convolution problem for only one of the array sensors.

In the time domain, the output at the ith-array sensor amounts to the convolution of a data stream $x_{i}(n)$ and the weight vector $\tilde{w}_{i}=\left[w_{i, 1}, \ldots, w_{i, Q}\right]^{\mathrm{T}}$. To study the effect of decimation, we consider a block of input data expressed by a vector $\tilde{x}_{i}(n)=\left[x_{i}(n), \ldots, x_{i}(n-M+1)\right]^{\mathrm{T}}$, where $M \geq Q$. Since the weight vector is updated independently in each block, we adopt the overlap-save method, rather than the overlapadd method [17]. ${ }^{1}$ Overlap-add method can also be used for frequency-domain processing, but it requires special attention, since it adds up convolution results of different blocks within which the weight vector may assume different values

\footnotetext{
${ }^{1}$ The concepts underlying the overlap-save and overlap-add methods are given in $[28,29]$. The use of these two methods in subband signal processing is discussed in [17].
}
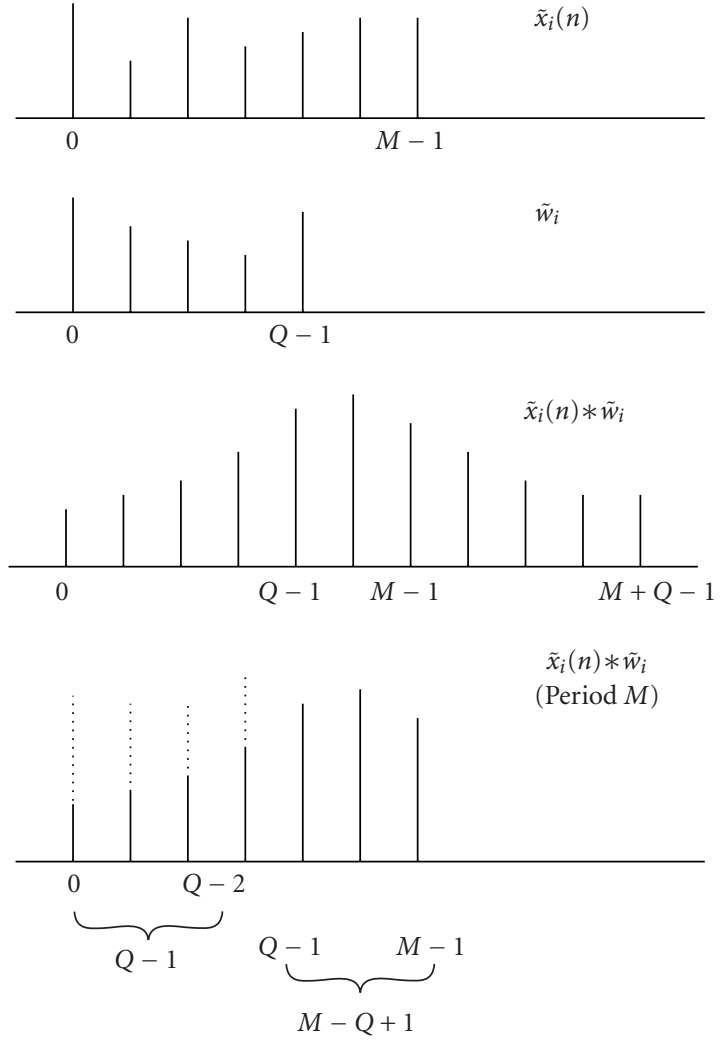

Figure 1: Illustration of alias problem (“*” denotes the convolution operator).

[30]. Referring to Figure 1, the convolution of $\tilde{w}_{i}$ and $\tilde{x}_{i}(n)$ yields a new vector of length $M+Q-1$, of which, only $M-Q+1$ samples (from the $Q$ th sample to the $M$ th sample) take full consideration of $Q$ data inputs. The rest are incomplete, in the sense that the output samples do not use all $Q$ input data. In this case, zero-padded data are used instead.

When DFT-based filter banks are used to construct a subband array, the data vector, along with the weight vector, is transformed into the subband domain. After the data vector and the weight vector are multiplied in the transform domain, the result is transformed back to the time domain by using the inverse DFT (IDFT).

For the unconstrained subband array structure, the length of both data and weight vectors is equal to the dimension of DFT. When we perform the convolution of the $M \times 1$ data vector and the $M \times 1$ weight vector, the result is a vector of length $2 M-1$. Therefore, when the $M$-point convolution is obtained from the IDFT of the product of the DFTs of the data and weight vectors, the first $M-1$ samples are contaminated by alias, and only the last sample is alias-free. As a result, in order to avoid alias, the only choice is $L=1$, that is, no decimation is made, which is the case considered in [7]. When $L>1$, alias problem arises and performance degradation occurs. For the centralized feedback scheme, the alias is controlled such that the error over the $L$ samples of the output data is minimized. 
It is noticed that the weight vector can be constrained such that, in each virtual channel, only the first $Q$ values of its time-domain equivalence are nonzero, where $Q \leq M$. In the constrained subband arrays, the lengths of data and weight vectors as well as the dimension of DFT can be different. With the use of an $M$-point DFT transform, the convolution of $M$-tap data and $Q$-tap weights yields $M-Q+1$ points of alias-free output samples. That is, the decimation rate can take the value $L \leq M-Q+1$ without causing an alias problem. It is pointed out that the alias-free results are achieved at the cost of reduced number of degrees of freedom from $M$ to $Q$, which, as we will show later, does not necessarily improve the system performance.

\section{SUBBAND ARRAYS}

\subsection{Formulation of subband array signals}

In this section, we formulate the expression of a DFT-based subband array with $M$ subbands and a decimation factor of $L$. Let the subband decomposition divide the $M$ samples of data sequence at the output of the $i$ th virtual channel,

$$
\tilde{x}_{i}(n)=\left[x_{i}(n), \ldots, x_{i}(n-M+1)\right]^{\mathrm{T}},
$$

into $M$ subbands, that is, to form the vector

$$
\tilde{x}_{T, i}(n)=\left[x_{i}^{(1)}(n), \ldots, x_{i}^{(M)}(n)\right]^{\mathrm{T}},
$$

where $i=1, \ldots, N J$, and the superscript $(m)$ denotes the data component at the $m$ th subband. $\tilde{x}_{i}(n)$ and $\tilde{x}_{T, i}(n)$ are related by the following equation:

$$
\tilde{x}_{T, i}(n)=\left[x_{T, i}^{(1)}(n), x_{T, i}^{(2)}(n), \ldots, x_{T, i}^{(M)}(n)\right]^{\mathrm{T}}=\mathbf{T}_{o} \tilde{x}_{i}(n),
$$

where $\mathbf{T}_{o}$ is the $M \times M$ DFT matrix with its $(i, k)$ th element being $\left[\mathbf{T}_{o}\right]_{i, k}=(1 / \sqrt{M}) W_{M}^{(i-1)(k-1)}, i, k=1, \ldots, M$, and $W_{M}=\exp (-j 2 \pi / M)$. It is noted that $\mathbf{T}_{o}$ is unitary and symmetric, that is, $\mathbf{T}_{o} \mathbf{T}_{o}^{\mathrm{H}}=\mathbf{T}_{o}^{\mathrm{H}} \mathbf{T}_{o}=\mathbf{I}_{M}$ and $\mathbf{T}_{o}^{\mathrm{T}}=\mathbf{T}_{o}$, where $\mathbf{I}_{M}$ is the $M \times M$ identity matrix. Then, the $N J \times 1$ data vector at the $m$ th subband is obtained as

$$
\begin{aligned}
\underline{\mathrm{x}}_{T}^{(m)}(n)= & {\left[x_{T, 1}^{(m)}(n), x_{T, 2}^{(m)}(n), \ldots, x_{T, N J}^{(m)}(n)\right]^{\mathrm{T}} } \\
= & {\left[\begin{array}{c}
\underline{\mathrm{x}}_{1}^{\mathrm{T}}(n) \\
\underline{\mathrm{x}}_{2}^{\mathrm{T}}(n) \\
\vdots \\
\underline{\mathrm{x}}_{N J}^{\mathrm{T}}(n)
\end{array}\right]\left[\begin{array}{c}
W_{M}^{0} \\
W_{M}^{m-1} \\
\vdots \\
W_{M}^{(m-1)(M-1)}
\end{array}\right] . }
\end{aligned}
$$

By defining

$$
\mathbf{x}_{T}(n)=\left[\left(\underline{\mathbf{x}}_{T}^{(1)}(n)\right)^{\mathrm{T}}, \ldots,\left(\underline{\mathbf{x}}_{T}^{(M)}(n)\right)^{\mathrm{T}}\right]^{\mathrm{T}}
$$

as the $M N J \times 1$ signal vector for all the $M$ subbands in the subband array, we can relate $\mathbf{x}_{T}(n)$ and $\mathbf{x}(n)$, defined in (5), by

$$
\mathbf{x}_{T}(n)=\mathbf{T} \mathbf{x}(n),
$$

where the transform matrix $\mathrm{T}$ is expressed in the form

$$
\mathbf{T}=\mathbf{T}_{o} \otimes \mathbf{I}_{N J}
$$

and $\otimes$ denotes the Kronecker product operator. It is easy to confirm that $\mathbf{T}$ is also unitary, that is, $\mathbf{T T}^{\mathrm{H}}=\mathbf{T}^{\mathrm{H}} \mathbf{T}=\mathbf{I}_{M N J}$.

\subsection{Adaptive subband arrays}

Denote by $\left(\underline{\mathbf{w}}_{T}^{(m)}\right)^{*}$ the $N J \times 1$ weight vector to the signal vector $\underline{\mathbf{x}}_{T}^{(m)}(n)$ at the $m$ th subband, and by $\mathbf{w}_{T}^{*}=\left[\left(\underline{\mathbf{w}}_{T}^{(1)}\right)^{\mathrm{T}}, \ldots\right.$, $\left.\left(\underline{\mathbf{w}}_{T}^{(M)}\right)^{\mathrm{T}}\right]^{\mathrm{H}}$ the $M N J \times 1$ weight vector to the entire subband signal vector $\mathbf{x}_{T}(n)$. The subband output is obtained as the following $M \times 1$ vector:

$$
\tilde{\mathbf{y}}_{T}(n)=\left[\begin{array}{c}
\left(\underline{\mathbf{w}}_{T}^{(1)}\right)^{\mathrm{H}} \underline{\mathbf{x}}_{T}^{(1)}(n) \\
\vdots \\
\left(\underline{\mathbf{w}}_{T}^{(M)}\right)^{\mathrm{H}} \underline{\mathbf{x}}_{T}^{(M)}(n)
\end{array}\right]=\mathbf{X}_{T}^{\mathrm{T}}(n) \mathbf{w}_{T}^{*},
$$

where

$$
\mathbf{X}_{T}(n)=\left[\begin{array}{ccc}
\underline{\mathbf{x}}_{T}^{(1)}(n) & & \mathbf{0} \\
& \ddots & \\
\mathbf{O} & & \underline{\mathbf{x}}_{T}^{(M)}(n)
\end{array}\right]
$$

is an $M N J \times M$ matrix. The time-domain output is the last $L$ interested samples out of the $M$ samples of the IDFT of $\tilde{\mathbf{y}}_{T}(n)$, expressed as

$$
\tilde{\mathbf{y}}(n)=\mathbf{U}_{L} \mathbf{T}_{o}^{-1} \tilde{\mathbf{y}}_{T}(n)=\mathbf{U}_{L} \mathbf{T}_{o}^{-1} \mathbf{X}_{T}^{\mathrm{T}}(n) \mathbf{w}_{T}^{*}=\mathbf{X}^{\mathrm{T}}(n) \mathbf{w}_{T}^{*},
$$

where

$$
\mathbf{U}_{L}=\left[\begin{array}{cc}
\mathbf{O}_{(M-L) \times(M-L)} & \mathbf{O}_{(M-L) \times L} \\
\mathbf{O}_{L \times(M-L)} & \mathbf{I}_{L}
\end{array}\right]
$$

is an $M \times M$ mask matrix with $\mathbf{O}_{a \times b}$ denoting the $a \times b$ zero matrix, and the $M N J \times M$ matrix

$$
\mathbf{X}(n)=\mathbf{X}_{T}(n) \mathbf{T}_{o}^{-1} \mathbf{U}_{L}
$$

is defined for notational convenience. Note in (25) that $\tilde{\mathbf{y}}(n)$ has only $L$ nonzero elements and the results of other $M-L$ symbols of the block are evaluated at other blocks. 


\section{Unconstrained subband arrays}

We first consider the unconstrained subband array structure. To help the derivation, we consider the following weight update equation based on the LMS algorithm:

$$
\mathbf{w}_{T} \longleftarrow \mathbf{w}_{T}+\mu \mathbf{X}_{T}(n) \tilde{\mathbf{e}}_{T}^{*}(n),
$$

where $\mu$ is a scalar representing the step size, ${ }^{2}$ and $\tilde{\mathbf{e}}_{T}(n)$ is the $M \times 1$ error signal vector at the transform domain. As we discuss below, the error signal vector is different for the two different feedback schemes. To avoid confusion, notations $\tilde{\mathbf{e}}_{T, \mathrm{CF}}(n)$ and $\tilde{\mathbf{e}}_{T, \mathrm{LF}}(n)$ will be used to specify the centralized and localized feedback schemes, respectively, for the error vector in the transform domain $\tilde{\mathbf{e}}_{T}(n)$.

In the centralized feedback scheme, for each block of subband array processing, the error between the reference signal and the subband array output is minimized in the time domain over $L$ samples, that is, ${ }^{3}$

$$
\begin{aligned}
\tilde{\mathbf{e}}(n) & =\mathbf{U}_{L} \tilde{\mathbf{s}}_{1}(n-v)-\mathbf{U}_{L} \mathbf{T}_{o}^{-1} \tilde{\mathbf{y}}_{T}(n) \\
& =\mathbf{U}_{L}\left[\tilde{\mathbf{s}}_{1}(n-v)-\mathbf{T}_{o}^{-1} \mathbf{X}_{T}^{\mathrm{T}}(n) \mathbf{w}_{T}^{*}\right] \\
& =\mathbf{U}_{L} \tilde{\mathbf{s}}_{1}(n-v)-\mathbf{X}^{\mathrm{T}}(n) \mathbf{w}_{T}^{*}
\end{aligned}
$$

The corresponding error vector at the subband domain $\tilde{\mathbf{e}}_{T, \mathrm{CF}}(n)$ is the DFT of the time-domain error and is expressed as

$$
\tilde{\mathbf{e}}_{T, \mathrm{CF}}(n)=\mathbf{T}_{o} \tilde{\mathbf{e}}(n)=\mathbf{T}_{o} \mathbf{U}_{L}\left[\tilde{\mathbf{s}}_{1}(n-v)-\mathbf{T}_{o}^{-1} \mathbf{X}_{T}^{\mathrm{T}}(n) \mathbf{w}_{T}^{*}\right]
$$

where

$$
\tilde{\mathbf{s}}_{1}(n)=\left[\begin{array}{lllll}
s_{1}(n+M-L) & \cdots & s_{1}(n) & \cdots & s_{1}(n-L+1)
\end{array}\right]^{\mathrm{T}}
$$

is a block of $M$ symbol values of the reference signal.

On the other hand, for the localized feedback scheme, the error between the reference signal and the subband array output is minimized independently at each subband. The error signal vector $\tilde{\mathbf{e}}_{T}(n)$ becomes

$$
\begin{aligned}
\tilde{\mathbf{e}}_{T, \mathrm{LF}}(n) & =\tilde{\mathbf{s}}_{T}(n-v)-\tilde{\mathbf{y}}_{T}(n) \\
& =\mathbf{T}_{o} \tilde{\mathbf{s}}_{1}(n-v)-\mathbf{X}_{T}^{\mathrm{T}}(n) \mathbf{w}_{T}^{*} .
\end{aligned}
$$

By comparing (30) and (32), it is evident that, while the centralized feedback scheme minimizes the error over the $L$ samples, the localized feedback scheme minimizes the error at all the $M$ samples independent of the decimation rate. In particular, when $L=M$, that is, the subband array is maximally decimated, $\mathbf{U}_{L}=\mathbf{I}_{M}$ and, subsequently, $\tilde{\mathbf{e}}_{T, \mathrm{CF}}(n)=\tilde{\mathbf{e}}_{T, \mathrm{LF}}(n)$. Therefore, the centralized and localized feedback schemes have the identical performance when the subband arrays are maximally decimated.

\footnotetext{
${ }^{2}$ The selection of step size is discussed in Section 7.

${ }^{3}$ It is noted that, although the same notation is used for the STAP system and different subband array schemes, the optimum value of $v$ could differ in different implementations, even under the same signal environment.
}

\section{Constrained subband arrays}

For constrained subband arrays, the weight vector is updated according to

$$
\mathbf{w}_{T} \longleftarrow \mathbf{w}_{T}+\mu \mathbf{F X}_{T}(n) \tilde{\mathbf{e}}_{T}^{*}(n)
$$

where

$$
\mathbf{F}=\left(\mathbf{T}_{o} \mathbf{U}_{Q} \mathbf{T}_{o}^{-1}\right) \otimes \mathbf{I}_{N J}=\mathbf{T U}_{Q N J} \mathbf{T}^{-1}
$$

is used to convert the transform-domain information into the time domain, mask the weights to only $Q$ nonzero values $(L=M-Q+1)$, and then convert the results back to the transform domain, with

$$
\begin{gathered}
\mathbf{U}_{Q}=\left[\begin{array}{cc}
\mathbf{I}_{Q} & \mathbf{O}_{Q \times(M-Q)} \\
\mathbf{O}_{(M-Q) \times Q} & \mathbf{O}_{(M-Q) \times(M-Q)}
\end{array}\right], \\
\mathbf{U}_{Q N J}=\left[\begin{array}{cc}
\mathbf{I}_{Q N J} & \mathbf{O}_{Q N J \times(M-Q) N J} \\
\mathbf{O}_{(M-Q) N J \times Q N J} & \mathbf{O}_{(M-Q) N J \times(M-Q) N J}
\end{array}\right] .
\end{gathered}
$$

It is clear that, under the same DFT transform dimensionality, the constrained subband array algorithm achieves aliasfree convolution at the cost of sacrificing the degrees-offreedom of the independently controllable weights.

For the constrained subband array structure, (29)-(32) remain valid with the understanding that not every element of $\mathbf{w}_{T}$ can be independently optimized.

\section{STEADY-STATE PERFORMANCE ANALYSIS}

This section derives the expressions of the steady-state MMSE performance. The performance of the unconstrained subband array structure is derived in Section 5.1, whereas, that of the constrained structure is derived in Section 5.2.

\subsection{Unconstrained subband arrays}

We first consider the performance of the centralized feedback subband arrays. From (28) and the orthogonality principle, $E\left[\mathbf{X}_{T}(n) \tilde{\mathbf{e}}_{T, C F}^{*}(n)\right]=\mathbf{0}$ at the steady state. Note that $\mathbf{T}_{o}^{\mathrm{H}}=\mathbf{T}_{o}^{-1}$ and $\mathbf{T}_{o}^{\mathrm{T}}=\mathbf{T}_{o}$, and therefore,

$$
\begin{aligned}
E\left[\mathbf{X}_{T}\right. & \left.(n) \tilde{\mathbf{e}}_{T, \mathrm{CF}}^{*}(n)\right] \\
& =E\left[\mathbf{X}_{T}(n) \mathbf{T}_{o}^{*} \mathbf{U}_{L}\left(\tilde{\mathbf{s}}_{1}^{*}(n-v)-\left(\mathbf{T}_{o}^{-1}\right)^{*} \mathbf{X}_{T}^{\mathrm{H}}(n) \mathbf{w}_{T}\right)\right] \\
& =M E\left[\mathbf{X}(n) \tilde{\mathbf{s}}_{1}^{*}(n-v)-\mathbf{X}(n) \mathbf{X}^{\mathrm{H}}(n) \mathbf{w}_{T}\right] \\
& =M\left(\mathbf{r}-\mathbf{R} \mathbf{w}_{T}\right)=\mathbf{0},
\end{aligned}
$$

where

$$
\begin{gathered}
\mathbf{R}=E\left[\mathbf{X}(n) \mathbf{X}^{\mathrm{H}}(n)\right], \\
\mathbf{r}=E\left[\mathbf{X}(n) \tilde{\mathbf{s}}_{1}^{*}(n-v)\right] .
\end{gathered}
$$


The optimum weight vector is the Wiener-Hopf solution

$$
\mathbf{w}_{T, \mathrm{CF}, \mathrm{opt}}=\mathbf{R}^{-1} \mathbf{r} \text {. }
$$

Using the above equation and (29), it is straightforward to obtain the MMSE of the time-domain output error:

$$
\begin{aligned}
\operatorname{MMSE}_{\mathrm{CF}} & =\frac{1}{L} E\left[\tilde{\mathbf{e}}^{\mathrm{H}}(n) \tilde{\mathbf{e}}(n)\right] \\
& =\frac{1}{L} E\left[\tilde{\mathbf{s}}_{1}^{\mathrm{H}}(n) \mathbf{U}_{L} \tilde{\mathbf{s}}_{1}(n)-\mathbf{r}^{\mathrm{H}} \mathbf{R}^{-1} \mathbf{r}\right] \\
& =1-\frac{1}{L} \mathbf{r}^{\mathrm{H}} \mathbf{R}^{-1} \mathbf{r} .
\end{aligned}
$$

Similarly, for the localized feedback scheme,

$$
\begin{aligned}
E\left[\mathbf{X}_{T}\right. & \left.(n) \tilde{\mathbf{e}}_{T, \mathrm{LF}}^{*}(n)\right] \\
& =E\left[\mathbf{X}_{T}(n) \mathbf{T}_{o}^{*} \tilde{\mathbf{s}}_{1}^{*}(n-v)-\mathbf{X}_{T}(n) \mathbf{X}_{T}^{\mathrm{H}}(n) \mathbf{w}_{T}\right] \\
& =\mathbf{r}_{T}-\mathbf{R}_{T} \mathbf{w}_{T}=\mathbf{0},
\end{aligned}
$$

where

$$
\begin{gathered}
\mathbf{R}_{T}=E\left[\mathbf{X}_{T}(n) \mathbf{X}_{T}^{\mathrm{H}}(n)\right], \\
\mathbf{r}_{T}=E\left[\mathbf{X}_{T}(n) \mathbf{T}_{o}^{*} \widetilde{\mathbf{s}}_{1}^{*}(n-v)\right] .
\end{gathered}
$$

Therefore, the optimum weight vector is

$$
\mathbf{w}_{T, \mathrm{LF}, \mathrm{opt}}=\mathbf{R}_{T}^{-1} \mathbf{r}_{T}
$$

and the corresponding MMSE is obtained as

$$
\begin{aligned}
\operatorname{MMSE}_{\mathrm{LF}} & =\frac{1}{L} E\left[\tilde{\mathbf{e}}^{\mathrm{H}}(n) \tilde{\mathbf{e}}(n)\right] \\
& =1+\frac{1}{L}\left[\mathbf{r}_{T}^{\mathrm{H}} \mathbf{R}_{T}^{-1} \mathbf{R}_{T}^{-1} \mathbf{r}_{T}-2 \operatorname{Re}\left(\mathbf{r}_{T}^{\mathrm{H}} \mathbf{R}_{T}^{-1} \mathbf{r}\right)\right]
\end{aligned}
$$

where $\operatorname{Re}(\cdot)$ denotes the real-part operator.

It can be shown that, when $L=1$, that is, when there is no decimation, the MMSE of a centralized feedback subband array is the same as the MMSE of the corresponding STAP system $[7,15]$. Compared with a subband array using the centralized feedback scheme, a subband array with the localized feedback scheme provides inferior performance when $L<M$, and the performance of the two feedback schemes becomes identical when $L=M$. In this case, $\mathbf{R}_{T}=\mathbf{R}, \mathbf{r}_{T}=\mathbf{r}$, and (39) is identical to (43).

\subsection{Constrained subband arrays}

To derive the steady-state performance of the constrained subband arrays, we premultiply (33) by $\mathbf{T}^{-1}$. Using expression (34), we obtain the following weight update equation in the time-domain equivalence:

$$
\mathbf{T}^{-1} \mathbf{w}_{T} \longleftarrow \mathbf{T}^{-1} \mathbf{w}_{T}+\mu \mathbf{U}_{Q N J} \mathbf{T}^{-1} \mathbf{X}_{T}(n) \tilde{\mathbf{e}}_{T}^{*}(n) .
$$

Define

$$
\begin{aligned}
& \mathcal{X}(n)= \\
& {\left[\begin{array}{ccccc}
\tilde{\mathbf{x}}(n) & \tilde{\mathbf{x}}(n-M+1) & \cdots & \tilde{\mathbf{x}}(n-2) & \tilde{\mathbf{x}}(n-1) \\
\tilde{\mathbf{x}}(n-1) & \tilde{\mathbf{x}}(n) & \cdots & \tilde{\mathbf{x}}(n-3) & \tilde{\mathbf{x}}(n-2) \\
\vdots & \vdots & \ddots & \vdots & \vdots \\
\tilde{\mathbf{x}}(n-M+2) & \tilde{\mathbf{x}}(n-M+3) & \cdots & \tilde{\mathbf{x}}(n) & \tilde{\mathbf{x}}(n-M+1) \\
\tilde{\mathbf{x}}(n-M+1) & \tilde{\mathbf{x}}(n-M+2) & \cdots & \tilde{\mathbf{x}}(n-1) & \tilde{\mathbf{x}}(n)
\end{array}\right] .}
\end{aligned}
$$

It can be shown that [31]

$$
\mathbf{X}_{T}(n)=\frac{1}{\sqrt{M}} \mathbf{T} \mathcal{X}(n) \mathbf{T}_{o}^{-1}
$$

Substituting (46) in (44) yields

$$
\begin{gathered}
\mathbf{T}^{-1} \mathbf{w}_{T} \longleftarrow \mathbf{T}^{-1} \mathbf{w}_{T}+\frac{\mu}{\sqrt{M}} \mathbf{U}_{Q N J} \chi(n) \mathbf{T}_{o}^{-1} \tilde{\mathbf{e}}_{T}^{*}(n) \\
=\mathbf{T}^{-1} \mathbf{w}_{T}+\frac{\mu}{\sqrt{M}} \chi_{Q}(n) \mathbf{T}_{o}^{-1} \tilde{\mathbf{e}}_{T}^{*}(n),
\end{gathered}
$$

where $\mathcal{X}_{Q}(n)=\mathbf{U}_{Q N J} \mathcal{X}(n)$. The upper $Q N J \times M$ elements of $\mathcal{X}_{Q}(n)$ are equal to those of $\mathcal{X}(n)$, whereas, the other matrix elements are zero. From (47), it is clear that, at the steady state,

$$
E\left[\mathcal{X}_{Q}(n) \mathbf{T}_{o}^{-1} \tilde{\mathbf{e}}_{T}^{*}(n)\right]=0
$$

is satisfied.

Denote by $\tilde{\mathbf{w}}_{T}$ the weight vector before the constraints, that is, $\mathbf{w}_{T}=\mathbf{F} \tilde{\mathbf{w}}_{T}$. For the centralized feedback scheme, we obtain

$$
\begin{aligned}
E & {\left[\mathcal{X}_{Q}(n) \mathbf{T}_{o}^{-1} \tilde{\mathbf{e}}_{T}^{*}(n)\right] } \\
& =E\left\{\mathbf{U}_{Q N J} \mathcal{X}(n) \mathbf{T}_{o}^{-1} \mathbf{T}_{o}^{*} \mathbf{U}_{L}\left[\tilde{\mathbf{s}}_{1}(n-v)-\mathbf{T}_{o}^{-1} \mathbf{X}_{T}^{\mathrm{T}}(n) \mathbf{w}_{T}^{*}\right]^{*}\right\} \\
& =\sqrt{M} E\left[\mathbf{G}(n) \mathbf{U}_{L} \tilde{\mathbf{s}}_{1}^{*}(n-v)-\mathbf{G}(n) \mathbf{U}_{L} \mathbf{G}^{\mathrm{H}}(n) \mathbf{T}^{-1} \tilde{\mathbf{w}}_{T}\right] \\
& =\sqrt{M}\left[\mathbf{r}_{Q}-\mathbf{R}_{Q} \mathbf{T}^{-1} \tilde{\mathbf{w}}_{T}\right]=0,
\end{aligned}
$$

where

$$
\begin{gathered}
\mathbf{G}(n)=\mathbf{U}_{Q N J} \mathbf{T}^{-1} \mathbf{X}_{T}(n) \mathbf{T}_{o}^{-1}, \\
\mathbf{R}_{Q}=E\left[\mathbf{G}(n) \mathbf{U}_{L} \mathbf{G}^{\mathrm{H}}(n)\right], \\
\mathbf{r}_{Q}=E\left[\mathbf{G}(n) \mathbf{U}_{L} \tilde{\mathbf{s}}_{1}^{*}(n-v)\right] .
\end{gathered}
$$

Notice that $\mathbf{R}_{Q}$ is of rank $Q N J$ and, therefore, is rank deficient if $Q<M$. We define the following matrix pseudoinversion:

$$
\mathbf{R}_{Q}^{\#}=\sum_{i=1}^{Q N J} \lambda_{i}^{-1} \mathbf{u}_{i} \mathbf{u}_{i}^{\mathrm{H}}
$$

where $\lambda_{i}, i=1, \ldots, Q N J$, are the QNJ nonzero eigenvalues of $\mathbf{R}_{Q}$, and $\mathbf{u}_{i}$ are the eigenvectors corresponding to $\lambda_{i}$. 
Then, the optimum weight vectors are obtained from (49) as

$$
\begin{gathered}
\tilde{\mathbf{w}}_{T, \mathrm{CF}, \mathrm{opt}}=\mathbf{T R}_{\mathrm{Q}}^{\#} \mathbf{r}_{\mathrm{Q}}, \\
\mathbf{w}_{T, \mathrm{CF}, \mathrm{opt}}=\mathbf{F} \tilde{\mathbf{w}}_{T, \mathrm{CF}, \mathrm{opt}}=\mathbf{T U}_{\mathrm{QNJ}} \mathbf{R}_{\mathrm{Q}}^{\#} \mathbf{r}_{\mathrm{Q}} .
\end{gathered}
$$

The error signal vector for constrained centralized feedback subband arrays is given by

$$
\begin{aligned}
\tilde{\mathbf{e}}(n) & =\mathbf{U}_{L} \tilde{\mathbf{s}}_{1}(n-v)-\mathbf{X}^{\mathrm{T}}(n) \mathbf{w}_{T}^{*} \\
& =\mathbf{U}_{L} \tilde{\mathbf{s}}_{1}(n-v)-\mathbf{X}^{\mathrm{T}}(n) \mathbf{T}^{*} \mathbf{U}_{Q N J}\left(\mathbf{R}_{Q}^{*}\right)^{\#} \mathbf{r}_{Q}^{*} \\
& =\mathbf{U}_{L} \tilde{\mathbf{s}}_{1}(n-v)-\mathbf{U}_{L} \mathbf{G}^{\mathrm{T}}\left(\mathbf{R}_{Q}^{*}\right)^{\#} \mathbf{r}_{Q}^{*},
\end{aligned}
$$

and the MMSE is given by

$$
\mathrm{MMSE}_{\mathrm{CF}}=1-\frac{1}{L} \mathbf{r}_{\mathrm{Q}}^{\mathrm{H}} \mathbf{R}_{Q}^{\#} \mathbf{r}_{\mathrm{Q}} .
$$

For the localized feedback scheme,

$$
\begin{aligned}
E\left[\mathcal{X}_{Q}(n) \mathbf{T}_{o}^{-1} \mathbf{e}_{T}^{*}(n)\right] & \\
& =E\left\{\mathbf{U}_{Q N J} \mathcal{X}(n) \mathbf{T}_{o}^{-1}\left[\mathbf{T}_{o}^{*} \tilde{\mathbf{s}}_{1}(n-v)-\mathbf{X}_{T}^{\mathrm{T}}(n) \mathbf{w}_{T}^{*}\right]^{*}\right\} \\
& =\sqrt{M} E\left[\mathbf{G}(n) \tilde{\mathbf{s}}_{1}^{*}(n-v)-\mathbf{G}(n) \mathbf{G}^{\mathrm{H}}(n) \mathbf{T}^{-1} \tilde{\mathbf{w}}_{T}\right] \\
& =\sqrt{M}\left[\mathbf{r}_{Q}^{\prime}-\mathbf{R}_{Q}^{\prime} \mathbf{T}^{-1} \tilde{\mathbf{w}}_{T}\right]=0,
\end{aligned}
$$

where

$$
\begin{gathered}
\mathbf{R}_{Q}^{\prime}=E\left[\mathbf{G}(n) \mathbf{G}^{\mathrm{H}}(n)\right], \\
\mathbf{r}_{Q}^{\prime}=E\left[\mathbf{G}(n) \tilde{\mathbf{s}}_{1}^{*}(n-v)\right] .
\end{gathered}
$$

The optimum weight vectors are obtained as

$$
\begin{gathered}
\tilde{\mathbf{w}}_{T, \mathrm{LF}, \mathrm{opt}}=\mathbf{T}\left(\mathbf{R}_{\mathrm{Q}}^{\prime}\right)^{\#} \mathbf{r}_{\mathrm{Q}}^{\prime}, \\
\mathbf{w}_{T, \mathrm{LF}, \mathrm{opt}}=\mathbf{F} \tilde{\mathbf{w}}_{T, \mathrm{LF}, \mathrm{opt}}=\mathbf{T U}_{\mathrm{QNJ}}\left(\mathbf{R}_{Q}^{\prime}\right)^{\#} \mathbf{r}_{\mathrm{Q}}^{\prime},
\end{gathered}
$$

where $\left(\mathbf{R}_{Q}^{\prime}\right)^{\#}$ is the pseudoinversion of $\mathbf{R}_{Q}^{\prime}$, which is also of rank QNJ. The error signal vector for constrained localized feedback subband arrays is given by

$$
\begin{aligned}
\tilde{\mathbf{e}}(n) & =\mathbf{U}_{L} \tilde{\mathbf{s}}_{1}(n-v)-\mathbf{X}^{\mathrm{T}}(n) \mathbf{w}_{T}^{*} \\
& =\mathbf{U}_{L} \tilde{\mathbf{s}}_{1}(n-v)-\mathbf{X}^{\mathrm{T}}(n) \mathbf{T}^{*} \mathbf{U}_{Q N J}\left(\mathbf{R}_{Q}^{\prime *}\right)^{\#} \mathbf{r}_{Q}^{\prime *} \\
& =\mathbf{U}_{L} \tilde{\mathbf{s}}_{1}(n-v)-\mathbf{U}_{L} \mathbf{G}^{\mathrm{T}}\left(\mathbf{R}_{Q}^{\prime *}\right)^{\#} \mathbf{r}_{Q}^{\prime *} .
\end{aligned}
$$

The corresponding MMSE is obtained as

$$
\begin{aligned}
& \operatorname{MMSE}_{\mathrm{LF}} \\
& \quad=1+\frac{1}{L}\left[\mathbf{r}_{Q}^{\prime} \mathrm{H}\left(\mathbf{R}_{Q}^{\prime}\right)^{\#} \mathbf{R}_{Q}\left(\mathbf{R}_{Q}^{\prime}\right)^{\#} \mathbf{r}_{Q}^{\prime}-2 \operatorname{Re}\left(\mathbf{r}_{Q}^{\mathrm{H}}\left(\mathbf{R}_{Q}^{\prime}\right)^{\#} \mathbf{r}_{Q}^{\prime}\right)\right] .
\end{aligned}
$$

When $Q=M$, a constrained subband array is equal to its unconstrained counterpart. Similar to the unconstrained subband array cases, it can be readily shown that, when $L=$ $M$, we have $\mathbf{R}_{Q}^{\prime}=\mathbf{R}_{Q}, \mathbf{r}_{Q}^{\prime}=\mathbf{r}_{Q}$, and (54) and (59) become identical.

\section{DMI IMPLEMENTATION AND COMPUTATIONAL COSTS}

In this section, we consider the computational costs when the direct matrix inversion (DMI) implementation is applied. We use the number of complex multiplication operations as the measure of the computational cost. The unconstrained subband array structures are considered below. Assuming that the pseudomatrix inversion in (51) consumes roughly the same amount of computations as those of matrix inversion, the constrained structures require additional computations of $2 N J M$-point FFT to perform the weight masking.

When the DMI algorithm is used, the weight vectors for STAP and the centralized and localized feedback subband arrays are computed using the Wiener-Hopf solutions given by (9), (38), and (42), respectively, with the covariance matrices and correlation vectors being replaced by the corresponding estimates obtained from a block of data samples [32]. The dimension of all covariance matrices is $M N J \times M N J$.

We focus on the computational costs of computing the weight vector from the Wiener-Hopf solutions, and that for the DFT/IDFT operations required for the subband arrays. The Wiener-Hopf solution is equivalent to the Yule-Walker equation with a general right-hand side. With some modification to the Levison-Durbin recursions developed for YuleWalker equation, the computation of the weight vector from the Wiener-Hopf solution, for a $p \times p$ covariance matrix case, requires $O\left(4 p^{2}\right)$ complex multiplications ${ }^{4}$ [34].

However, for the localized feedback subband array, the covariance matrix $\mathbf{R}_{T}$ is block diagonal. To illustrate the corresponding computational requirements, we consider in the manner that the weight vector is updated at each subband independently. The weight vector $\underline{\mathbf{w}}_{T, \mathrm{LF}, \mathrm{opt}}^{(m)}$ at the $m$ th subband is obtained from

$$
\underline{\mathbf{w}}_{T, \mathrm{LF}, \mathrm{opt}}^{(m)}=\left(\mathbf{R}_{T}^{(m)}\right)^{-1} \mathbf{r}_{T}^{(m)},
$$

where

$$
\mathbf{R}_{T}^{(m)}=E\left[\left(\underline{\mathbf{x}}_{T}^{(m)}(n)\right)^{\mathrm{H}} \underline{\mathbf{x}}_{T}^{(m)}(n)\right]
$$

is the $N J \times N J$ covariance matrix of $\underline{x}_{T}^{(m)}$ at the $m$ th subband, and

$$
\mathbf{r}_{T}^{(m)}=E\left[\underline{\mathbf{x}}_{T}^{(m)}(n)\left(s^{(m)}(n)\right)^{*}\right]
$$

is the correlation vector between $\underline{x}_{T}^{(m)}$ and the reference signal $s^{(m)}(t)$ at the $m$ th subband.

Therefore, for the subband array using the localized feedback scheme, the weight vector can be obtained from $M$ parallel sets of dimension $\mathrm{NJ} \times \mathrm{NJ}$ matrix problems.

\footnotetext{
${ }^{4}$ There are algorithms for solving such equations which require just $O\left(p \log ^{2}(p)\right)$ operations. However, in a typical problem, the Levison-Durbin recursion is still the faster method due to the fact that these new algorithms require excessive codes [33].
} 
From the above discussion, it is clear that the computational cost of STAP system is $O\left(4 M^{2} N^{2} J^{2}\right)$. For the centralized feedback subband array, the computational cost is $O\left(4 M^{2} N^{2} J^{2}\right)$ per $L$ symbols, resulting in $O\left(4 M^{2} N^{2} J^{2} / L\right)$ flops per symbol. On the other hand, for the localized feedback subband array, the computational cost is $O\left(4 M N^{2} J^{2}\right)$ per $L$ symbols, resulting in $O\left(4 M N^{2} J^{2} / L\right)$ flops per symbol. In particular, when $L=M$, the computational cost for the centralized and localized feedback subband arrays are $O\left(4 M N^{2} J^{2}\right)$ and $O\left(4 N^{2} J^{2}\right)$, respectively.

For subband arrays, one must consider the computational cost of DFT/IDFT transforms. For every $L$ symbols, $N J$ times of $M$-dimensional DFT transforms are required at the subband signal decomposition, one time DFT is needed for reference signal decomposition, and one time IDFT is required for the signal synthesis at the subband array output. Therefore, the computational cost becomes $O((N)+$ 2) $\left.(M / L) \log _{2} M\right)=O\left((M N J / L) \log _{2} M\right)$ per symbol for both subband array schemes. Therefore, the computational cost of DFT/IDFT transforms is smaller than that of weight computations for the centralized feedback scheme, whereas for the localized feedback scheme, it becomes smaller than that of the weight computations only when $\log _{2} M<4 N J$, which is often satisfied.

\section{CONVERGENCE PERFORMANCE}

In this section, we consider the convergence performance of the subband arrays. The LMS algorithm is used. To take the advantages of subband array processing for improved convergence, we perform self-orthogonalization of the data signals in each subband independently after the subband decomposition [6]. Because the number of the virtual channels $(N J)$ is usually much smaller than that of the total STAP dimensions $(M N J)$, the additional computational cost of eigendecomposition at each subband is considerably lower than that of the whole-band subspace approach of subband array or STAP systems [21]. Note that, while power normalization is effective in improving the convergence performance in single-antenna equalizers, the effect of power normalization alone is not significant in subband arrays [6].

Consider the $k$ th subband, and let $\mathbf{R}_{T}^{(k)}$ denote the $N J \times$ $N J$ covariance matrix of subband signal vector $\underline{x}_{T}^{(k)}(n)$, and is eigendecomposed as

$$
\mathbf{R}_{T}^{(k)}=E\left[\underline{\mathbf{x}}_{T}^{(n)}(t)\left(\underline{\mathbf{x}}_{T}^{(k)}(n)\right)^{\mathrm{H}}\right]=\mathbf{V}^{(k)} \boldsymbol{\Lambda}^{(k)}\left(\mathbf{V}^{(k)}\right)^{\mathrm{H}} .
$$

The new subband signal vector after the self-orthogonalization is expressed as

$$
\underline{\underline{x}}_{T}^{(k)}(n) \longleftarrow\left(\boldsymbol{\Lambda}^{(k)}\right)^{-1 / 2}\left(\mathbf{V}^{(k)}\right)^{\mathrm{H}} \underline{\mathbf{x}}_{T}^{(k)}(n) .
$$

In practice, the covariance matrix $\mathbf{R}_{T}^{(k)}$ can be approximated using sample averaging or recursive update. Note that, while such data self-orthogonalization makes the comparison more obvious, it is common for all the subband array schemes and does not favor any specific scheme in the convergence performance comparison.

We first consider the unconstrained subband array using the centralized feedback scheme. The mean of the weight error vector can be expressed as [32]

$$
E\left[\mathbf{w}_{T}(l)-\mathbf{w}_{T, \mathrm{opt}}\right]=\left[1-\mu \mathbf{R}_{S}\right] E\left[\mathbf{w}_{T}(0)-\mathbf{w}_{T, \mathrm{opt}}\right],
$$

where $\mathbf{w}_{T}(l)$ denotes the subband-domain weight vector at the $l$ th iteration. From (28) and (30), the matrix $\mathbf{R}_{S}$ is obtained as follows:

(A) centralized, unconstrained:

$$
\mathbf{R}_{S}=E\left[\mathbf{X}_{T}(n) \mathbf{T}_{o}^{*} \mathbf{U}_{L}\left(\mathbf{T}_{o}^{*}\right)^{-1} \mathbf{X}_{T}^{\mathrm{H}}(n)\right] .
$$

Therefore, the step size is chosen as

$$
0<\mu=\frac{\alpha}{\operatorname{tr}\left(\mathbf{R}_{S}\right)}<\frac{2}{\operatorname{tr}\left(\mathbf{R}_{S}\right)},
$$

where $0<\alpha<2$ is a constant and $\operatorname{tr}(\cdot)$ denotes the matrix trace. From (28) and (30)-(33), it is straightforward to derive the matrix $\mathbf{R}_{S}$ for other subband array schemes in the similar manner as follows:

(B) localized, unconstrained:

$$
\mathbf{R}_{S}=E\left[\mathbf{X}_{T}(n) \mathbf{X}_{T}^{\mathrm{H}}(n)\right] ;
$$

(C) centralized, constrained:

$$
\mathbf{R}_{S}=E\left[\mathbf{F X}_{T}(n) \mathbf{T}_{o}^{*} \mathbf{U}_{L}\left(\mathbf{T}_{o}^{*}\right)^{-1} \mathbf{X}_{T}^{\mathrm{H}}(n)\right] ;
$$

(D) localized, constrained:

$$
\mathbf{R}_{S}=E\left[\mathbf{F X}_{T}(n) \mathbf{X}_{T}^{\mathrm{H}}(n)\right] .
$$

For the unconstrained subband array with the localized feedback scheme, the weights are updated independently at each subband. In this case, the step-size parameter at the $m$ th subband can be chosen as

$$
0<\mu^{(m)}=\frac{\alpha}{\operatorname{tr}\left(\mathbf{R}_{S}^{(m)}\right)}<\frac{2}{\operatorname{tr}\left(\mathbf{R}_{S}^{(m)}\right)},
$$

where

(E) localized, unconstrained:

$$
\mathbf{R}_{S}^{(m)}=E\left[\underline{\mathbf{x}}_{T}^{(m)}(n)\left(\underline{\mathbf{x}}_{T}^{(m)}(n)\right)^{\mathrm{H}}\right] .
$$

In the underlying case, due to self-orthogonalization, it follows that $\operatorname{tr}\left(\mathbf{R}_{S}^{(m)}\right)=(1 / M) \operatorname{tr}\left(\mathbf{R}_{S}\right)$ and, therefore, the step-size parameter $\mu^{(m)}$, obtained from (71), is $M$ times larger than $\mu$ obtained from (67). For constrained subband array with localized feedback scheme, extensive computer simulations have shown that the step-size parameter can be equally increased, leading to faster convergence. 


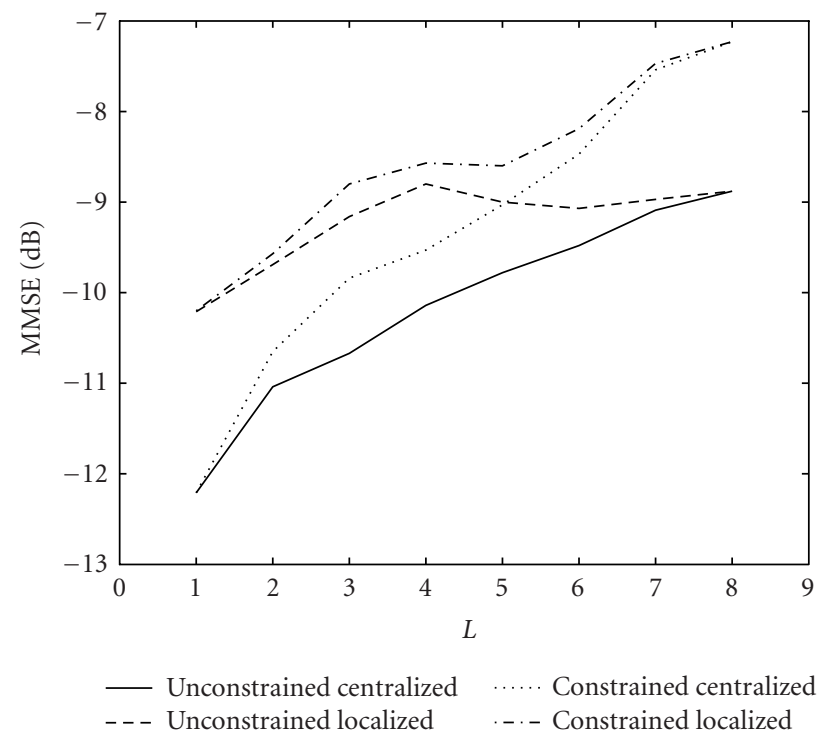

(a)

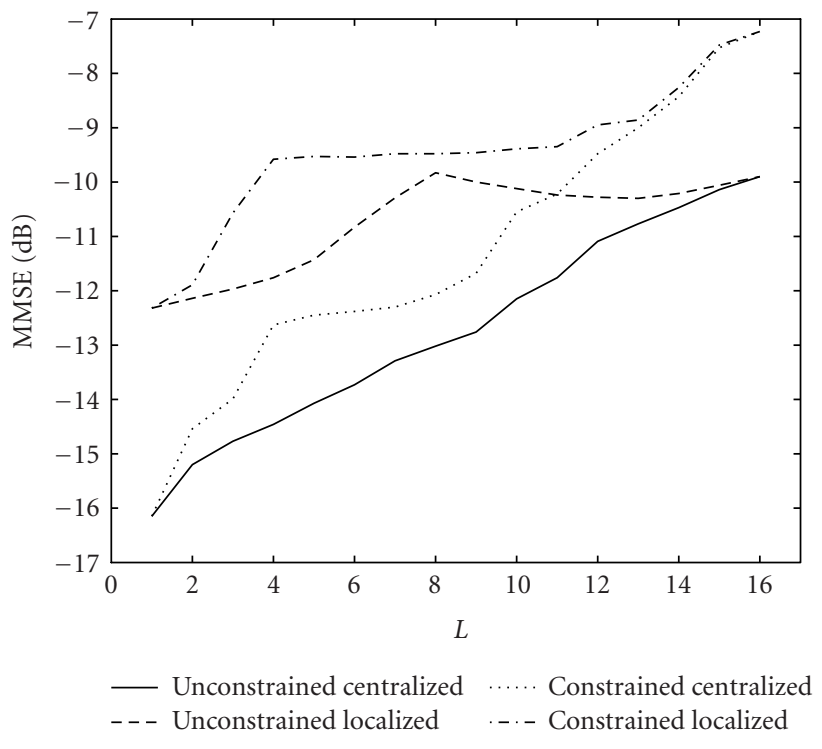

(b)

Figure 2: MMSE versus the decimation rate: (a) $M=8$ and (b) $M=16$.

\section{NUMERICAL EXAMPLES}

\subsection{Steady-state performance}

A three-element linear array with half-wavelength interelement spacing is considered. Two user signals are illuminating the array. Each has a maximum delay spread of five symbols. Six quasistatic multipath components are randomly generated for each user. The quasistatic channels are assumed to remain constant over the processing period, and to change over time as independent stationary stochastic processes. The mean value of the input SNR is $20 \mathrm{~dB}$ for both signals. For each user signal, the input signal power is defined as the total power of all paths. The signals are sampled and processed at the symbol rate (i.e., $J=1$ ).

DMI-like methods are considered in the performance evaluation. One hundred frequency-domain data samples are used for weight and MMSE computations, and the results are averaged over 100 independent trials.

Figure 2 shows the MMSE performance, where $M$ takes the values of 8 and 16, and $L$ assumes a value between 1 and $M$. For the constrained subband array structure, the number of nonzero weight elements is chosen as $Q=M-L+1$ in each virtual channel. It is evident that the 16-subband array provides lower MMSE than the 8 -subband counterpart. While the change shown in Figure 2 is not monotonic due to limited data samples used in the simulations, we maintain that the MMSE generally increases with $L$.

Among the four schemes of subband arrays, the results show that unconstrained subband array structures outperform the respective constrained counterparts, and the centralized feedback scheme provides superior performance compared to the localized feedback scheme. As a result, when the same values of $M$ and $L$ are considered, the un- constrained centralized feedback scheme achieves the best performance, whereas, the constrained localized feedback scheme provides the worst performance.

It is clear from the numerical results that, unlike other structures, the performance of the unconstrained subband arrays with the localized feedback scheme does not change significantly with respect to the decimation rate. This is because the weights are optimized in the frequency domain and, therefore, the subband array does not favor any timedomain samples in a subband block. For the constrained subband arrays, the MSE becomes large as the decimation rate increases, because the number of nonzero weights $Q=M-L+1$ decreases as the decimation rate $L$ increases.

While the decimation compromises the subband array performance, it is however noted, that the use of decimation often greatly reduces the signal processing rate and computational costs. The localized feedback scheme can further reduce the implementation complexity and it is amenable to parallel implementations. Therefore, the subband arrays in general provide flexible system designs, where performance may be traded off with the system complexity. Subband arrays with decimation and localized feedback schemes may, therefore, provide improved performance to the STAP system with the same computational costs.

\subsection{Convergence performance}

Next, we present the convergence performance of the subband arrays. Comparison between different subband array schemes are made and the effect of decimation rates are investigated. The array and signal parameters are the same as those used in steady-state performance computations. In all the simulations, $\alpha=0.4$ is used and the initial values of all 


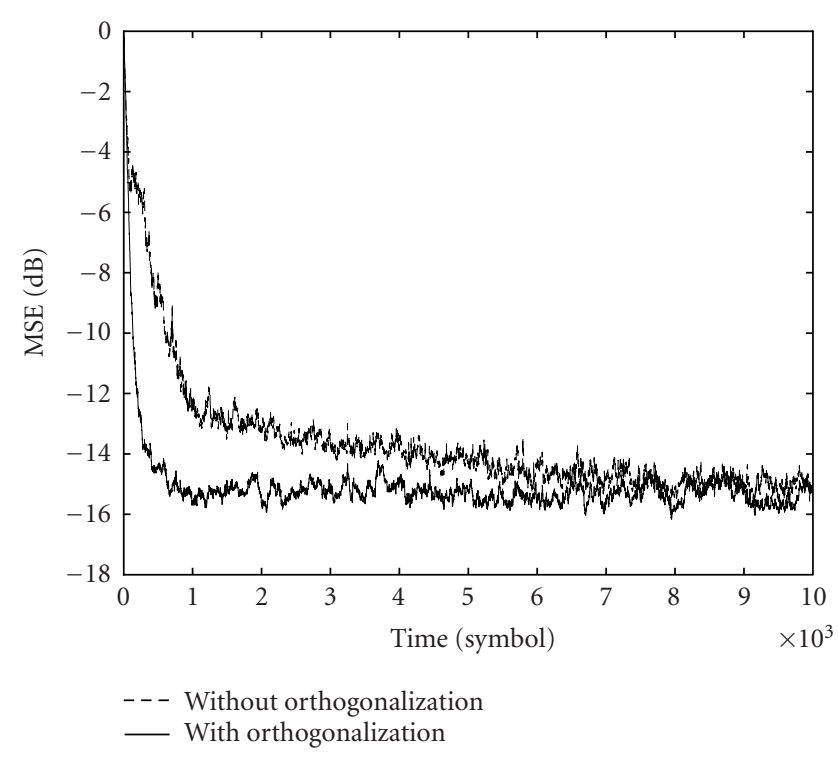

(a)

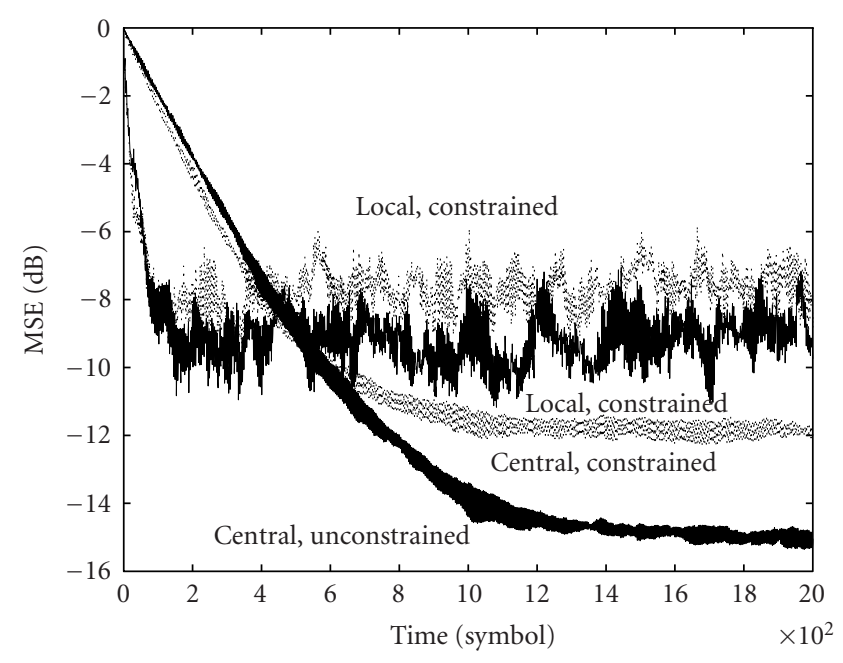

(c)

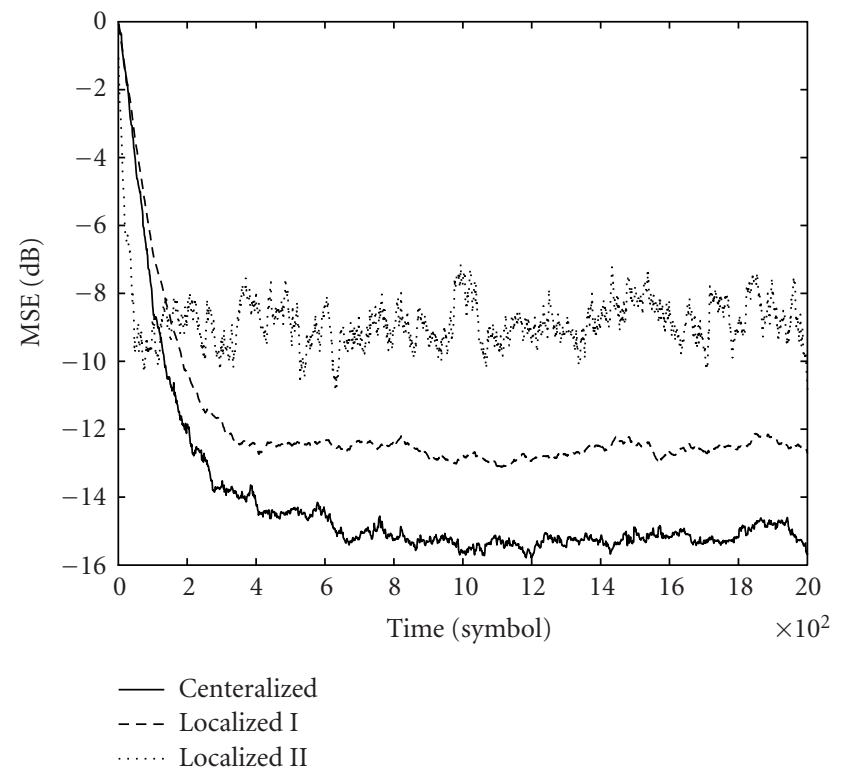

(b)

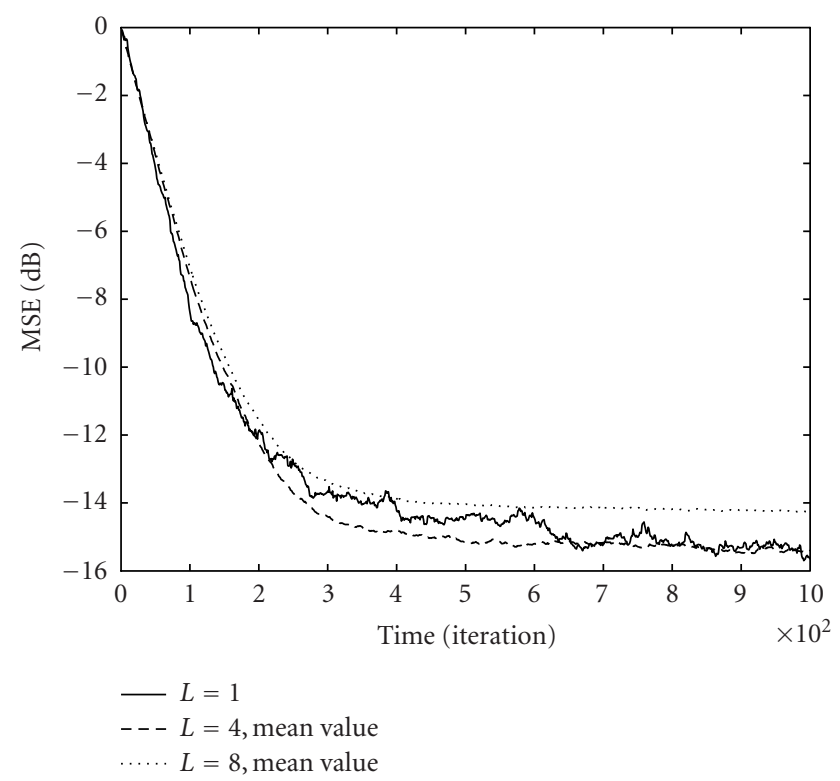

(d)

FIGURE 3: Comparison of the convergence performance for different subband array schemes. (a) Effect of self-orthogonalization (centralized, $M=16, L=1)$. (b) Convergence performance $(M=16, L=1)$. (c) Convergence performance with respect to symbols $(M=16, L=4)$. (d) Convergence performance with respect to iterations (centralized, unconstrained, $M=16$ ).

weights are set to zero. In addition, the MSE is obtained by averaging the results of 100 independent trials.

We first show the effect of data self-orthogonalization at each subband. The subband array with centralized feedback scheme is used as the example, and the localized feedback scheme is demonstrated in a similar manner. Figure 3a compares the convergence performance with and without data self-orthogonalization, where the number of subbands is $M=16$ and no decimation is applied $(L=1)$.
Note that, when no decimation is made, the constrained and unconstrained subband array schemes are identical. The conventional performance of a STAP system is the same as that of the subband array with no decimation and no data selforthogonalization. At the expense of eigendecompositions and multiplication of sixteen $3 \times 3$ matrices, the improvement of convergence performance using the self-orthogonalization is evident. In the rest of simulations, self-orthogonalized data are used. 
Figure $3 \mathrm{~b}$ shows the convergence performance of the subband arrays where no decimation is applied $(L=1)$. The constrained and unconstrained subband array schemes are identical. For the localized feedback subband array, if the selection of the step-size parameter $\mu$ is guided by (67) (shown as "localized I" in the figure), the convergence performance becomes similar to that of the centralized subband array. On the other hand, when the step-size parameter $M \mu$ is used for the localized feedback scheme (shown as "localized II"), the convergence becomes faster than the centralized counterpart, at the expense of larger residual error. Note that the centralized feedback subband array diverges when the step size is set equal to $M \mu$.

In Figure $3 c$, the convergence is compared for different subband array schemes for $L=4$. Step size $\mu$ is used for both centralized feedback schemes, whereas $M \mu$ is used for the localized feedback schemes. It is clear that, because of the reduction of the degrees of freedom, the constrained feedback schemes provide slightly faster convergence.

Comparing Figures $3 b$ and $3 c$, it is evident that the decimating of the data results in slower convergence. The primary reason behind this is that, in a decimated array, the weights are updated only every $L$ samples. In Figure $3 d$, the convergence performance is compared in terms of the number of iterations, and the convergence for different decimation rates $(L=1,4$, and 8$)$ are comparable.

\section{CONCLUSIONS}

We have investigated the MMSE performance of subband arrays with arbitrary decimation rates for unconstrained and constrained subband array structures. Both the centralized and localized feedback schemes were considered. Among the four combination schemes of subband arrays, the results showed that when the same number of array and subbands are used, the unconstrained subband array structures outperform the constrained counterparts, and the centralized feedback scheme provides superior performance compared to the localized feedback scheme.

\section{ACKNOWLEDGMENT}

The work of Y. Zhang and M. G. Amin is supported by the Office of Naval Research Grant no. N00014-98-1-0176.

\section{REFERENCES}

[1] A. J. Paulraj and C. B. Papadias, "Space-time processing for wireless communications," IEEE Signal Processing Magazine, vol. 14, no. 6, pp. 49-83, 1997.

[2] R. Kohno, "Spatial and temporal communication theory using adaptive antenna array," IEEE Personal Communications, vol. 5, no. 1, pp. 28-35, 1998.

[3] Y. Kamiya and Y. Karasawa, "Performance comparison and improvement in adaptive arrays based on the time and frequency domain signal processing," IEICE Transactions on Fundamentals of Electronics, Communications and Computer Sciences, vol. J82-A, no. 6, pp. 867-874, 1999.
[4] Y. Zhang, K. Yang, and Y. Karasawa, "Subband CMA adaptive arrays in multipath fading environment," Electronics and Communications in Japan (Part I: Communications), vol. 83, no. 11, pp. 43-54, 2000.

[5] Y. Zhang, K. Yang, and M. G. Amin, "Adaptive array processing for multipath fading mitigation via exploitation of filter banks," IEEE Trans. Antennas and Propagation, vol. 49, no. 4, pp. 505-516, 2001.

[6] Y. Zhang, K. Yang, and M. G. Amin, "Convergence performance of subband arrays for spatio-temporal equalization," in Proc. 11th IEEE Signal Processing Workshop on Statistical Signal Processing (SSP '01), pp. 544-547, Orchid Country Club, Singapore, August 2001.

[7] Y. Zhang, K. Yang, M. G. Amin, and Y. Karasawa, "Performance analysis of subband arrays," IEICE Transactions on Communications, vol. E84-B, no. 9, pp. 2507-2515, 2001.

[8] X. N. Tran, T. Taniguchi, and Y. Karasawa, "Performance analysis of subband adaptive array in multipath fading environment," IEICE Transactions on Fundamentals of Electronics, Communications and Computer Sciences, vol. E85-A, no. 8, pp. 1798-1806, 2002.

[9] Y. Zhang and K. Yang, "Subband adaptive arrays with different decimations," in Proc. IEEE Sensor Array and Multichannel Signal Processing Workshop (SAM '02), pp. 398-402, Rosslyn, Va, USA, August 2002.

[10] T. Sekiguchi and Y. Karasawa, "CMA adaptive array antennas using analysis and synthesis filter banks," IEICE Transactions on Fundamentals of Electronics, Communications and Computer Sciences, vol. E81-A, no. 8, pp. 1570-1577, 1998.

[11] A. O. Steinhardt and N. B. Pulsone, "Subband STAP processing, the fifth generation," in Proc. IEEE Sensor Array and Multichannel Signal Processing Workshop (SAM '00), pp. 1-6, Cambridge, Mass, USA, March 2000.

[12] J. M. Khalab and M. K. Ibrahim, "Novel multirate adaptive beamforming technique," Electronics Letters, vol. 30, no. 15, pp. 1194-1195, 1994.

[13] R. Hudson, D. Korompis, F. Lorenzelli, A. Wang, and K. Yao, "Subband processing for broad-band microphone arrays," Journal of VLSI Signal Processing Systems for Signal Image and Video Technology, vol. 14, no. 1, pp. 43-55, 1996.

[14] J. Benesty and D. R. Morgan, "Frequency-domain adaptive filtering revisited, generalization to the multi-channel case, and application to acoustic echo cancellation," in Proc. IEEE Int. Conf. Acoustics, Speech, Signal Processing (ICASSP '00), vol. 2, pp. II789-II792, Istanbul, Turkey, June 2000.

[15] R. T. Compton Jr., "The relationship between tapped delayline and FFT processing in adaptive arrays," IEEE Trans. Antennas and Propagation, vol. 36, no. 1, pp. 15-26, 1988.

[16] B. L. Lewis and F. F. Kretschmer Jr., "Naval research laboratory report of limited distribution," Tech. Rep., Naval Research Laboratory, Arlington, Va, USA, February 1974.

[17] J. J. Shynk, "Frequency-domain and multirate adaptive filtering," IEEE Signal Processing Magazine, vol. 9, no. 1, pp. 14-37, 1992.

[18] A. Feuer and R. Cristi, "On the steady state performance of frequency domain LMS algorithms," IEEE Trans. Signal Processing, vol. 41, no. 1, pp. 419-423, 1993.

[19] B. Rafaely and S. J. Elliot, "A computationally efficient frequency-domain LMS algorithm with constraints on the adaptive filter," IEEE Trans. Signal Processing, vol. 48, no. 6, pp. 1649-1655, 2000.

[20] R. M. M. Derkx, G. R. M. Egelmeers, and P. C. W. Sommen, "New constraining method for partitioned block frequencydomain adaptive filters," IEEE Trans. Signal Processing, vol. 50, no. 9, pp. 2177-2186, 2002. 
[21] K. Yang, Y. Zhang, and Y. Mizuguchi, "A signal subspacebased subband approach to space-time adaptive processing for mobile communications," IEEE Trans. Signal Processing, vol. 49, no. 2, pp. 401-413, 2001.

[22] K. Yang, Y. Zhang, and T. Ohira, "Array configuration design for space-time adaptive processing systems," in Proc. IEEE Sensor Array and Multichannel Signal Processing Workshop (SAM '02), pp. 145-148, Rosslyn, Va, USA, August 2002.

[23] A.-J. van der Veen, "Resolution limits of blind multi-user multi-channel identification schemes-the bandlimited case," in Proc. IEEE Int. Conf. Acoustics, Speech, Signal Processing (ICASSP '96), vol. 5, pp. 2722-2725, Atlanta, Ga, USA, May 1996.

[24] P. P. Vaidyanathan, Multirate Systems and Filter Banks, Prentice Hall, Englewood Cliffs, NJ, USA, 1993.

[25] H. Ochi, Y. Higa, and S. Kinjo, "A subband adaptive filter with the optimum analysis filter bank," in Proc. IEEE Int. Conf. Acoustics, Speech, Signal Processing (ICASSP '95), vol. 2, pp. 993-996, Detroit, Mich, USA, May 1995.

[26] D. Marelli and M. Fu, "Performance analysis for subband identification," IEEE Trans. Signal Processing, vol. 52, no. 1, pp. 142-154, 2004.

[27] T. Q. Nguyen, "Near-perfect-reconstruction pseudo-QMF banks," IEEE Trans. Signal Processing, vol. 42, no. 1, pp. 65-76, 1994.

[28] L. R. Rabiner and B. Gold, Theory and Application of Digital Signal Processing, Prentice Hall, Englewood Cliffs, NJ, USA, 1975.

[29] A. V. Oppenheim, R. W. Schafer, and J. R. Buck, DiscreteTime Signal Processing, Prentice Hall, Englewood Cliffs, NJ, USA, 2nd edition, 1999.

[30] G. A. Clark, S. R. Parker, and S. K. Mitra, “A unified approach to time- and frequency-domain realization of FIR adaptive digital filters," IEEE Trans. Acoustics, Speech, and Signal Processing, vol. 31, no. 5, pp. 1073-1083, 1983.

[31] R. M. Gray, "On the asymptotic eigenvalue distribution of Toeplitz matrices," IEEE Transactions on Information Theory, vol. 18, no. 6, pp. 725-730, 1972.

[32] S. Haykin, Adaptive Filter Theory, Prentice Hall, Englewood Cliffs, NJ, USA, 3rd edition, 1996.

[33] D. B. Percival and A. T. Walden, Spectral Analysis for Physical Applications: Multitaper and Conventional Univariate Techniques, Cambridge University Press, Cambridge, UK, 1993.

[34] G. H. Golub and C. F. van Loan, Matrix Computations, Johns Hopkins University Press, Baltimore, Md, USA, 3rd edition, 1996.

Yimin Zhang received the M.S. and Ph.D. degrees from the University of Tsukuba, Tsukuba, Japan, in 1985 and 1988, respectively. He joined the faculty of the Department of Radio Engineering, Southeast University, Nanjing, China, in 1988. He served as a Technical Manager at the Communication Laboratory Japan, Kawasaki, Japan, from 1995 to 1997, and was a Visiting Researcher at ATR Adaptive Communications

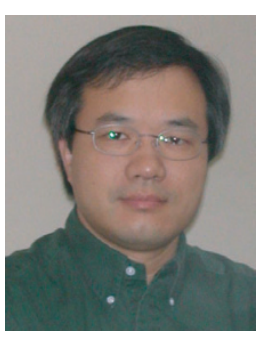
Research Laboratories, Kyoto, Japan, from 1997 to 1998. Since 1998, he has been with the Villanova University, Villanova, Pa, where he is currently a Research Associate Professor at the Center for Advanced Communications. His research interests are in the areas of array signal processing, space-time adaptive processing, multiuser detection, MIMO systems and cooperative diversity, blind signal processing, digital mobile communications, and timefrequency analysis. Dr. Zhang is a Senior Member of IEEE.
Kehu Yang received the B.E., M.S., and Ph.D. degrees from Xidian University (formerly, the Northwest Telecommunications Engineering Institute), Xi'an, China, in 1982, 1984, and 1995, respectively. $\mathrm{He}$ joined Xidian University in 1985, where he became an Associate Professor in May 1996. From December 1998 to May 2002, he was a Visiting Researcher at ATR Adaptive Communications Research Laboratories, Kyoto,

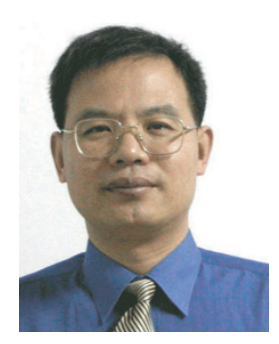
Japan. From June 2002 to October 2002, he was a Research Fellow at Xi'an Research Institute of ZTE Corporation. From November 2002 to December 2002, he was with Xi'an Haitian Antenna Technologies Co., Ltd., as the Leader of the R\&D Department 3. In January 2003, he rejoined Xidian University as an Associate Professor, and in July 2004, he became a Full Professor at Xidian University. His main research interests are in array signal processing for radar and smart antenna for mobile communications. Dr. Yang is a Member of IEEE.

Moeness G. Amin received his Ph.D. degree in 1984 from University of Colorado, Boulder. He has been on the faculty of Villanova University since 1985, where is now a Professor in the Department of Electrical and Computer Engineering and the Director of the Center for Advanced Communications. Dr. Amin has over 250 publications in the areas of wireless communications, time-frequency analysis, smart an-

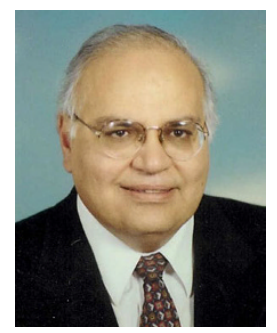
tennas, interference cancellation in broadband communication platforms, over-the-horizon radar, and channel equalizations. Dr. Amin was the Technical Chair of the IEEE International Symposium on Signal Processing and Information Technology, Pennsylvania, 2002; the General and Organization Chair of the IEEE Workshop on Statistical Signal and Array Processing, Pennsylvania, 2000; and the General and Organization Chair of the IEEE International Symposium on Time-Frequency and Time-Scale Analysis, Pennsylvania, 1994. He was an Associate Editor of the IEEE Transactions on Signal Processing during 1996-1998. Dr. Amin is a Fellow of the Institute of Electrical and Electronics Engineers (IEEE); the recipient of the IEEE Third Millennium Medal; Distinguished Lecturer of the IEEE Signal Processing Society for 2003; Member of the Franklin Institute Committee of Science and the Arts; and recipient of the 1997 Villanova University Outstanding Faculty Research Award. 